\title{
Linx
}

Revue des linguistes de l'université Paris X Nanterre

$51 \mid 2004$

Théories de l'écriture et pratiques scolaires

\section{Approche cognitive de l'activité rédactionnelle et de son acquisition. Le rôle de la mémoire de travail}

\section{Annie Piolat}

\section{OpenEdition}

\section{Journals}

Édition électronique

URL : http://journals.openedition.org/linx/174

DOI : 10.4000/linx.174

ISSN : 2118-9692

Éditeur

Presses universitaires de Paris Nanterre

\section{Édition imprimée}

Date de publication : 1 décembre 2004

Pagination : 55-74

ISSN : 0246-8743

\section{Référence électronique}

Annie Piolat, «Approche cognitive de l'activité rédactionnelle et de son acquisition. Le rôle de la mémoire de travail », Linx [En ligne], 51 | 2004, mis en ligne le 28 janvier 2011, consulté le 19 avril 2019. URL : http://journals.openedition.org/linx/174; DOI : 10.4000/linx.174 


\title{
Approche cognitive de l'activité rédactionnelle et de son acquisition. Le rôle de la mémoire de travail
}

\author{
Annie PiElat \\ Centre PsyCLÉ, EA 3273, Université de PrEvence \\ 29 avenue REbert-Schuman, 13621 Aix-en-PrEvence, Cedex 1, France \\ annie.piElat@up.univ-mrs.fr
}

Introduction: Pour questionner, chercher, et ne pas prescrire de pilule rédactionnelle

En près de 25 ans, l'étude des processus rédactionnels de textes a considérablement évolué. Les psycholinguistiques et/ou les psychologues du langage n'envisagent plus exactement leur objet d'étude dans les termes fixés par Hayes et Flower (1980). Durant ces années, les possibilités méthodologiques d'expérimentation ont été clairement amplifiées et renouvelées (pour une synthèse, cf. Olive, 2002 ; Piolat \& Pélissier, 1998), accompagnant et provoquant de nouvelles délimitations du champ de recherche (pour une synthèse, cf. Alamargot \& Chanquoy, 2002 ; Chanquoy \& Alamargot, 2002).

Dans le cadre de cet article, l'objectif est d'illustrer la place essentielle que la composante cognitive appelée «Mémoire de travail» a prise dans les modèles du rédacteur que celui-ci soit habile en écriture ou encore en apprentissage. Les quelques modèles auxquels il sera fait référence n'ont pas l'ambition de décrire exhaustivement le fonctionnement du rédacteur et ne constituent surtout pas des modèles «à suivre » ou «à apprendre» pour améliorer l'activité de production verbale écrite. Ces architectures cognitives circonscrivent la composition écrite de textes afin de favoriser l'élaboration d'heuristiques de recherche en psychologie cognitive et expérimentale. Elles sont, de ce fait, forcément limitées et limitatives. Les quelques éléments d'informations données ici sur les aspects fonctionnels de l'activité de rédaction des textes, ont donc comme simple mission d'informer, si nécessaire, linguistiques et didacticiens. Il s'agit de décrire, selon les termes de Garcia-Debanc et Fayol (2003), 
des lieux possibles de dialogue et de collaboration interdisciplinaires afin de pouvoir questionner la complexité de l'activité du rédacteur débutant ou non.

Tout d'abord, le rôle de la mémoire de travail dans les activités complexes et leur apprentissage sera présenté. Puis l'évolution des modélisations de l'activité rédactionnelle et celle de son acquisition seront abordées afin de souligner les nouveaux enjeux théoriques en rédaction de textes. Place sera ensuite faite aux architectures cognitives récentes de la production écrite qui intègrent explicitement la mémoire de travail. Une rapide illustration de l'intérêt pour la recherche de ce renouveau des modèles rédactionnels sera, enfin, proposée.

\section{La mémoire de travail, cheville ouvrière des activités cognitives complexes et de leur acquisition}

En langage quotidien, le terme de mémoire renvoie à une unique fonction de stockage mental de l'information. Les travaux de psychologie cognitive ont cependant mis en évidence une grande variété de représentations mentales et de processus. Avec le concept de mémoire à long terme, les psychologues font référence à des états mentaux stables concernant de vastes configurations d'informations alors qu'avec celui de mémoire à court terme, ils évoquent les états mentaux concernant le stockage très éphémère de quelques éléments. Avec celui de mémoire de travail, ils rendent compte d'un stockage temporaire mais non passif de l'information. Cette mémoire assurerait, en effet, plusieurs fonctions de contrôle (encore appelées fonctions exécutives) dans la prise en charge de situations nouvelles. De nombreux développements théoriques et expérimentaux sur ce thème concernent, à la fois, l'étude de la mémoire et de l'attention. Il en découle que les traitements de l'information sur lesquels les individus exercent un contrôle sont supposés nécessiter des ressources mentales (appelées également ressources attentionnelles). Aussi, le fonctionnement de la mémoire de travail est souvent comparé à celui d'un système de gestion de ressources mentales.

Les quelques éléments laconiques qui viennent d'être donnés ne doivent pas laisser penser que dans ce dynamique et vaste champ de recherche de la psychologie cognitive, les points de vue sont consensuels; les modèles et contre modèles des processus mnésiques font rage (pour des synthèses, cf. Gaonac'h \& Larigauderie, 2000). La conception de Baddeley $(1990,2000)$ a le mérite d'être très partagée et toujours source d'hypothèses fécondes de recherche alors que d'autres modèles sont encore très peu explorés.

\subsection{Le modèle de Baddeley (2000), un modèle toujours beuristique}

Baddeley (2000) rappelle avec malice que cela fait maintenant plus de 30 ans que sa conception de la mémoire de travail rend compte d'un nombre considérable de résultats expérimentaux et qu'elle reste à bien des égards pertinente pour expliquer certains aspects des traitements cognitifs. Par l'ajout de composants « cristallisés » (cf. Figure 1), cet auteur remanie son modèle princeps paru dès 1974, afin de rendre compte de la gestion en temps réel d'informations de formats différents et provenant de plusieurs sources. 


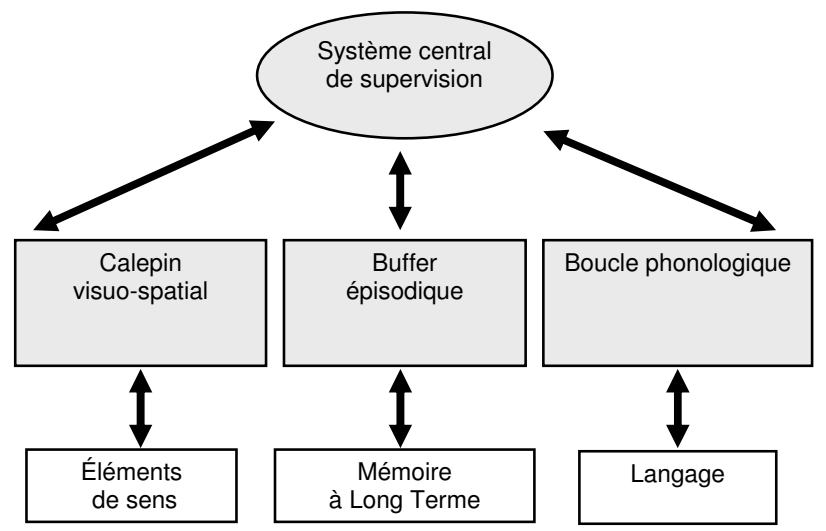

Figure 1. Les composants de la mémoire de travail d'après Baddeley (2000)

(Légende : en gris les composants flexibles, en blanc, les composants cristallisés).

Ce modèle peut être rapidement décrit comme suit. Le système central de supervision, appelé aussi administrateur central, remplit différentes fonctions : inhibition des réponses automatiques ou d'informations devenues non pertinentes, activation d'informations dans la mémoire à long terme, planification d'activité et attribution de ressources. Il s'agit donc de la composante attentionnelle de la mémoire de travail coordonnant, sélectionnant et contrôlant les opérations de traitement. Sous la dépendance de ce système central, les composants spécialisés (appelés aussi systèmes esclaves) que sont la boucle phonologique et le calepin visuo-spatial, stockent très temporairement, pour le premier, des informations verbales et, pour le second, des informations visuelles et spatiales. Si l'individu souhaite maintenir dans les systèmes esclaves des informations ou si le système cognitif doit maintenir des informations utiles à un traitement automatique, il procède à leur rafraîchissement par autorépétition mentale dans la boucle phonologique. Toujours, sous le contrôle de l'administrateur central, le buffer épisodique, lui aussi à capacité limitée et temporaire, permet de fédérer en une représentation intégrée, des informations conceptuelles, sémantiques, visuo-spatiales, phonétiques en provenance de la mémoire à long terme et/ou des deux systèmes esclaves. Il constitue ainsi une interface majeure de gestion des informations entre les systèmes esclaves et la mémoire à long terme. Ces différents éléments gèrent des informations qualifiées de flexibles parce qu'en constante mutation, alors que la mémoire à long terme fournit des éléments d'information stables, qualifiés de cristallisées.

Ainsi pour les psychologues de la cognition qui adhèrent à ce modèle, les individus disposent de structures de stockage temporaire de quelques informations (les systèmes esclaves), mais aussi d'unités de traitement (l'administrateur central et le buffer épisodique) qui concourent à la transformation de l'information. Comme le mettent en évidence d'abondantes expériences de laboratoire, les activités complexes comme la lecture-compréhension, la production écrite de textes, la résolution de problèmes, les activités mathématiques, l'apprentissage d'une seconde langue (etc.), sont sous la dépendance fonctionnelle d'une telle structure, que cette activité soit efficiente ou non (dysfonctionnement) et que l'individu soit ou non en situation d'apprentissage (pour une synthèse, cf. Gaonac'h \& Fradet, 2003). 
Cette conception de la mémoire de travail parait rendre équivalentes les notions de mémoire de travail et d'attention. Diverses propositions théoriques ont été avancées pour rendre compte de la gestion des ressources attentionnelles. Il s'agit de pouvoir analyser comment le système cognitif établit des priorités de traitements, ou encore instaure une certaine flexibilité dans ses plans de traitements. Baddeley (2000) rassemble dans le superviseur central et le buffer épisodique, la plupart des fonctions exécutives qui assurent cette flexibilité. Aussi, il ne semble vraiment pas opportun de distinguer, comme l'a fait Kellogg (1994 ; cf. section 3) dans son premier modèle de la rédaction de textes, un composant «Attention» et un composant «Mémoire de travail» pour renvoyer à ces fonctions de gestion des opérations cognitives. Elles seraient administrées par la mémoire de travail.

\subsection{Développement de la mâ̂trise rédactionnelle : encore une question de mémoire de travail}

De façon très générale, la gestion des ressources cognitives disponibles chez les individus peut être décrite comme suit. La mise en œuvre d'une activité complexe serait contrainte par la capacité limitée de ressources dont dispose l'individu. Cette capacité serait différente d'un individu à l'autre. Étant donnée cette limite, les individus ne peuvent conduire en parallèle qu'un nombre limité d'opérations car le coût de ces traitements ne peut dépasser les ressources attentionnelles disponibles. Pour rester dans le cadre imposé par la capacité et éviter toute "surcharge », il est efficace, via l'apprentissage, d'automatiser certains des traitements. Un traitement automatique est réalisé de façon involontaire, rapide et irrépressible, sans interférer avec d'autres traitements. Il n'implique pas le recours d'un contrôle conscient et donc une d'attention soutenue. Un traitement automatique ne mobilise pas ou peu l'administrateur central de la mémoire de travail et laisse «de la place » à d'autres traitements. En revanche, un traitement délibéré, plus lent à réaliser, est sous la dépendance fonctionnel de l'administrateur central. La réalisation de ce traitement est plus ou moins coûteuse selon sa nature.

Cette gestion des ressources cognitives a été validée pour la compréhension de textes. Elle est aussi été repérée par McCutchen (1996) lors de l'apprentissage de la production de textes par des rédacteurs débutants. Par hypothèse, la maitrise de la rédaction de texte correspondrait ainsi à la gestion coordonnée de traitements (peu coûteux quand ils peuvent être automatisés) et de traitements très demandeurs en ressources s'ils sont délibérés comme doivent l'être certaines opérations de planification et de révision de textes. Aussi, la mémoire de travail devrait jouer un rôle clé.

Toutefois, avant de traiter ce point, une question préalable doit être posée : la capacité de la mémoire de travail évolue-t-elle en fonction du développement de l'enfant ? La réponse n'est pas simple et ne peut être limitée à l'affirmation d'un accroissement de la capacité de la mémoire de travail au fils des âges et de la pratique (pour une synthèse, cf. Gaonac'h \& Larigauderie, 2000). Plusieurs déterminants de ce développement sont à l'étude: (1) accélération d'opérations fondamentales comme l'identification des éléments qui feront l'objet des traitements ; (2) amélioration de processus stratégiques parmi lesquels la possibilité d’utiliser la répétition mentale dans la boucle phonologique ; (3) amélioration des relations entre mémoire à court terme et mémoire de travail afin d'accroître le stock 
des connaissances disponibles pendant les traitements. Gaonac'h et Fradet (2003) rassemblent les travaux qui ont montré de manière plus spécifique comment les différents composants de la mémoire de travail (administrateur central, boucle phonologique, calepin visuo-spatial) étaient l'objet d'un développement, expliquant ainsi l'accroissement des performances dans différentes activités scolaires.

Pour ce qui concerne le développement même de la production écrite verbale, le rôle de la mémoire de travail et de ses fonctions exécutives commence à être maintenant bien repéré (pour une synthèse, cf. Bourdin, 2002). Comme le mettent en évidence les modèles de l'activité rédactionnelle présentés ci-après (sections 2 et 3), la production verbale écrite est une activité complexe qui implique au moins trois niveaux de traitement qui mobilisent des connaissances diversifiées (cf. Figure 2 pour une typologie de ces différentes connaissances) et des processus, outils de transformation de l'information (cf. sections 2 et 3 pour un descriptif).

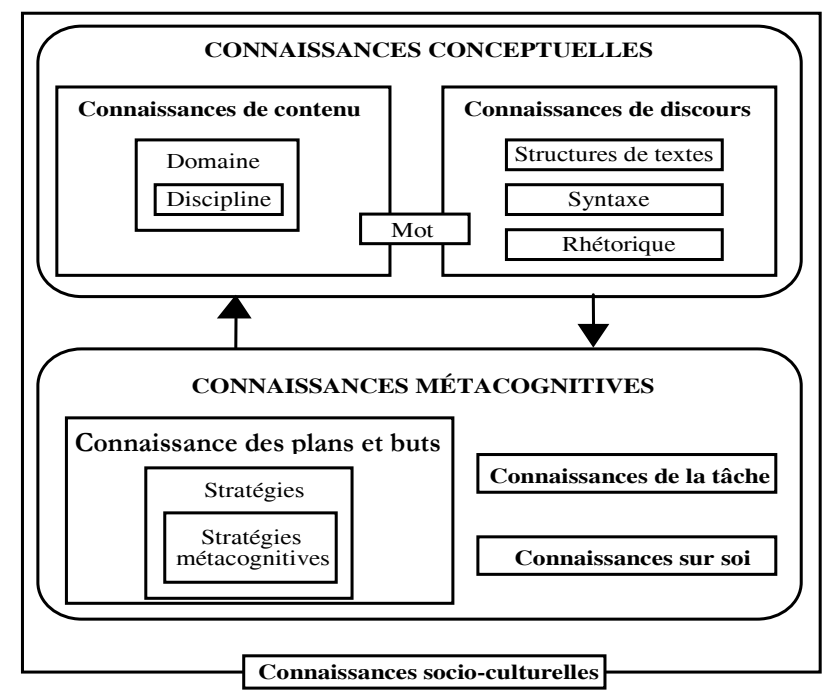

Figure 2. Catégorisation des connaissances selon Dolchy et Alexander (1995).

Le rédacteur doit construire un ensemble de représentations pré-verbales regroupant les idées qu'il veut communiquer dans un contexte donné (niveau sémantique). Ce message doit faire l'objet de traitements afin d'être mis en langue (niveau linguistique). Ces représentations verbales sont elles-mêmes transformées pour être transcrites (niveau grapho-moteur). C'est la maittrise fonctionnelle de chacun de ces niveaux mais aussi la gestion entre ces différents niveaux qui rendent l'acquisition de la rédaction de texte tellement complexe. En effet, les traitements impliqués par chacun de ces niveaux mobilisent d'importantes ressources attentionnelles en début d'apprentissage, condition même de l'acquisition. De plus, deux paramètres interviennent dans l'aptitude à les coordonner en temps réel: (1) l'efficience des traitements impliqués; (2) la capacité mnésique du rédacteur. Ce sont ces deux aspects qui doivent faire l'objet d'un apprentissage. Le jeune rédacteur, par l'entraînement, doit automatiser en priorité certains traitements comme l'exécution de lettres afin de pouvoir 
consacrer plus de ressources à des traitements non automatisables comme certaines opérations complexes de planification et de révision. Il doit aussi développer des procédures métacognitives de gestion de l'ensemble de son activité, procédures toujours exigeantes en ressources cognitives (cf. section 2.2.).

\section{Vingt-cinq ans déjà de modélisation du processus d'écriture : routes et déroute}

Il est impossible ici de faire l'historique des différents modèles rédactionnels en psychologie (cf. Alamargot \& Chanquoy, 2002 ; Piolat, 1990 ; Piolat \& Roussey, 1992). Il est plutôt question d'évoquer succinctement les modèles qui ont clairement intégré la mémoire de travail dans leur architecture cognitive. Chemin faisant, les chercheurs ont alors étonnamment complexifié (Hayes, 1996) ou simplifié (Kellogg, 1996) le modèle princeps de Hayes et Flower (1980). Les cheminements intellectuels des psychologues ont pris des routes parfois orthogonales aboutissant à des bornages de processus déroutants, sinon inattendus, si l'on compare la publication, la même année, de deux modèles précédemment cités (cf. section 3).

\subsection{Hayes et Flower (1980), le modèle fondateur toujours pas abandonné}

Hayes et Flower (1980) posent les premières définitions des différents processus rédactionnels disponibles chez le rédacteur adulte, définitions encore largement partagées par toute la communauté de chercheurs lorsqu'il s'agit de les évoquer rapidement. Le processus de planification permet de construire, au niveau conceptuel, un message pré-verbal correspondant aux idées que le rédacteur veut transmettre. Avec cet outil cognitif, le rédacteur récupère en mémoire à long terme des informations, les (re)-organise si besoin est, tout en élaborant des plans d'action. Le processus de traduction (encore appelé de mise en texte) lui permet de transformer les élaborations pré-verbales en un message verbal (encodage graphémique, orthographique, lexicale, syntaxique). Le processus de révision, outil de contrôle, favorise l'évaluation du texte produit (ou en cours d'élaboration) en le comparant à la représentation mentale concernant le texte souhaité. Il permet aussi d'examiner la formulation du texte en fonction des standards requis par les contraintes de la situation de communication. Sur un plan fonctionnel, ces différents composants sont actualisés autant de fois qu'il est nécessaire et selon la succession utile à chaque rédacteur. Cette mobilisation est d'ailleurs tellement différente selon les individus que Levy et Ransdell (1995) la qualifie de signature rédactionnelle.

Le rôle programmatique de ce modèle a été très conséquent. Les chercheurs se sont, en effet, évertués à mieux connaître les différents sous processus en jeu et les types de connaissances disponibles en mémoire à long terme et mobilisées par ces processus. Hayes et Flower ont d'ailleurs intégré les acquis expérimentaux découlant de la voie ainsi ouverte, en spécifiant de façon très féconde les sous processus de révision (Flower, Hayes, Carey, Schriver, \& Stratman, 1986) et ceux du processus de planification (pour des revues, voir Piolat, 1998, 1999). Ils ont alors statué avec une grande finesse sur les caractéristiques fonctionnelles de ces processus de traitement de l'information lorsque le rédacteur adulte planifie et contrôle ce qu'il écrit. Toutefois, aucun rôle fonctionnel n'a 
été alors accordé à la mémoire de travail. Seul le concept de capacité de stockage limitée est avancé, permettant de qualifier de surcharge soit la mobilisation d'un processus trop gourmand en ressources cognitives, soit la convocation de deux activités dont l'activation simultanée dépasse la capacité disponible du rédacteur.

Les limites de ce modèle princeps sont maintenant bien connues (Chanquoy \& Alarmargot, 2002). En termes de "chaînons manquants », Hayes et Flower et leur équipe de recherche, cantonnés à étudier les performances rédactionnelles des adultes chez lesquels l'activité graphique est fortement automatisée et donc très disponible, n’ont pas contribué à l'analyse des processus graphiques. Ils n'ont pas fait non plus de la mise en texte leur champ de recherche privilégié. Dans le même temps et grâce à des techniques de recherches en laboratoire très sophistiquées, Levelt (1986) a proposé un modèle de la production verbale orale capital pour comprendre comment les locuteurs réalisent la production d'énoncés. Les composants de son modèle sont les suivants. Outre le conceptualiseur qui assure la mise en place d'un message préverbal, le modèle comporte un formulateur qui procède à l'encodage grammatical et phonologique des éléments lexicaux sous forme de langage interne. Un articulateur prend en charge la réalisation physique de l'émission verbale. Ces composants sont coordonnés avec un système de comprébension du discours qui contrôle la production verbale orale. Sur le plan fonctionnel, ce contrôle qui peut être interne (c'est-à-dire avant l'émission verbale) ou externe (pendant et après l'émission verbale), ne peut être exercé que si le locuteur dispose de suffisamment de ressources. L'influence du modèle de Levelt (1986) dans le champ d'étude de la production verbale orale est considérable, champ pour lequel il a été conçu. Il a aussi été utilisé pour mieux appréhender la production verbale écrite dont certains aspects fonctionnels sont estimés communs avec l'oral (pour une synthèse, Fayol, 1997). Il a même fait l'objet d'une adaptation par van Wijk (1999) moyennant quelques modifications mineures (cf. Figure 3).

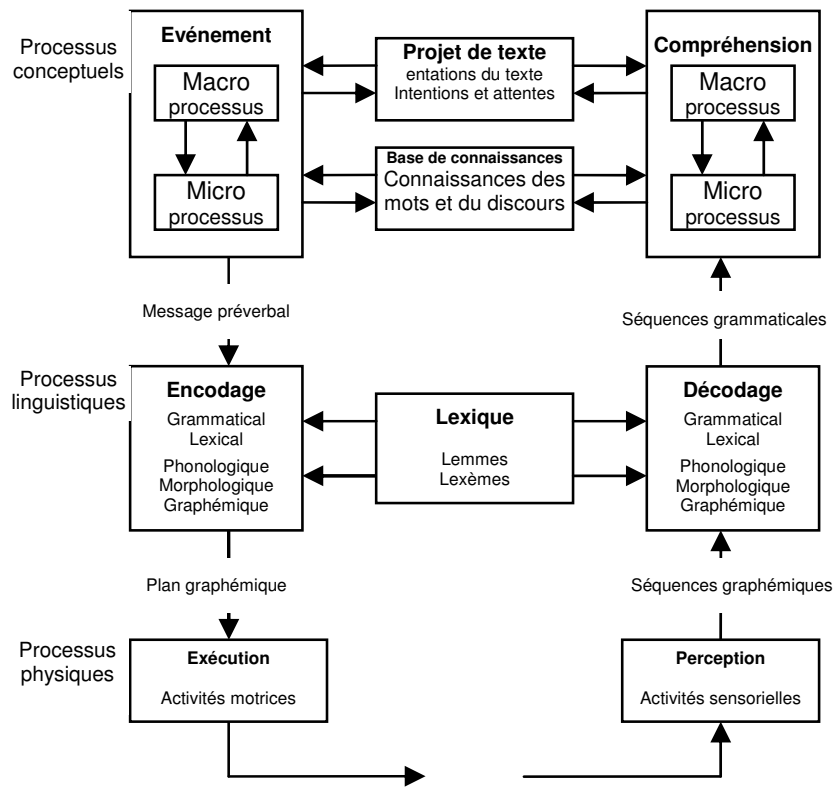

Figure 3. Modèle de production écrite inspiré de Levelt (1989) selon van Wijk (1999). 
Le modèle de van Wijk (1999) n'est pas inspiré par celui de Hayes et Flower (1980) même si le composant Processus conceptuels réunit des fonctions proches de la Planification et le composant Processus linguistiques englobe des fonctions proches de la Mise en texte. Mais, comme ce dernier, il ne fait pas référence explicitement à la mémoire de travail et à ses fonctions.

\section{2. Quand l'étude de l'évolution de la mâ̂trise rédactionnelle a un impact certain sur le modèle adulte princeps}

Chronologiquement, ce sont les chercheurs soucieux de rendre compte de l'évolution de la maîtrise rédactionnelle qui ont relevé l'importance du rôle de la mémoire de travail, celle-ci pouvant en partie expliquer fonctionnellement les contraintes développementales comme cela a été brièvement exposé ci avant (section 1.2.). Deux contributions majeures peuvent être évoquées, celle de Scarmadalia et Bereiter (1991) et celle de Berninger et Swanson (1994).

A partir de leurs observations, Scarmadalia et Bereiter (1991) décrivent deux stratégies d'utilisation des connaissances qui rendent compte des traitements opérés essentiellement lors de la planification par des rédacteurs novices et experts. La stratégie des connaissances racontées, principalement employée par les rédacteurs novices, consiste à récupérer une connaissance en mémoire à long terme et à la transcrire, le texte écrit servant de source d'activation pour la récupération de nouvelles connaissances qui sont à leur tour transcrites. Cette stratégie cyclique est guidée par les contraintes de la situation de production qui orientent la récupération des connaissances. Les textes ainsi produits sont des juxtapositions de phrases qui reflètent la structure des connaissances du rédacteur. Les rédacteurs plus experts ne se contentent pas d'une simple transcription de leurs connaissances. Ils les réorganisent pour les rendre compatibles avec les contraintes thématiques et rhétoriques imposées. Cette stratégie des connaissances transformées consiste ainsi dans la complexification du processus de planification. Acquise de façon progressive, elle serait disponible vers l'âge de 16 ans. Par la prise en compte de contraintes supplémentaires, les rédacteurs experts parviennent à rédiger des textes plus élaborés que ceux produits par les novices. De plus, par l'analyse qu'ils font de la situation de communication et par la réorganisation de leurs connaissances, les rédacteurs expérimentés acquièrent des compétences et des connaissances supplémentaires pendant qu'ils écrivent. Cet aspect épistémique de la stratégie des connaissances transformées constitue un point crucial de l'expertise rédactionnelle. Toutefois, celle-ci n'est pas la seule utilisée, les rédacteurs experts pouvant, dans des situations de productions familières, se contenter d'appliquer la stratégie des connaissances racontées. Plus focalisés sur les aspects stratégiques et dynamiques de l'activité que sur l'identification des processus rédactionnels, Scarmadalia et Bereiter (1991) ne décrivent pas ces stratégies rédactionnelles à partir du modèle de Hayes et Flower (1980). En revanche, ils notent que le passage de la première stratégie à la seconde dépend de l'augmentation avec l'âge des capacités de maintien et de traitement des informations en mémoire de travail.

Tout en enrichissant le modèle de Hayes et Flower (1980), Berninger et Swanson (1994) proposent, quant à eux, trois phases de développement (en termes de 
niveau scolaire) qui montrent à la fois le décalage entre la possible mobilisation des différents processus rédactionnels (intra-phase et inter-phases) mais aussi le rôle crucial de la mémoire de travail dans la deuxième et troisième phases. Pour l'essentiel, dans la première phase (cf. Figure 4), le processus de traduction permet au rédacteur débutant (6-7 ans) d'assurer en premier lieu la transcription graphémique (qui occupe largement la capacité de la mémoire de travail) suivie de celle de la production de mots, de phrases, de paragraphes, de textes. Le rôle du processus de planification est restreint à l'enchaînement de phrases, celui du processus de révision à des corrections de surface (orthographe et ponctuation).

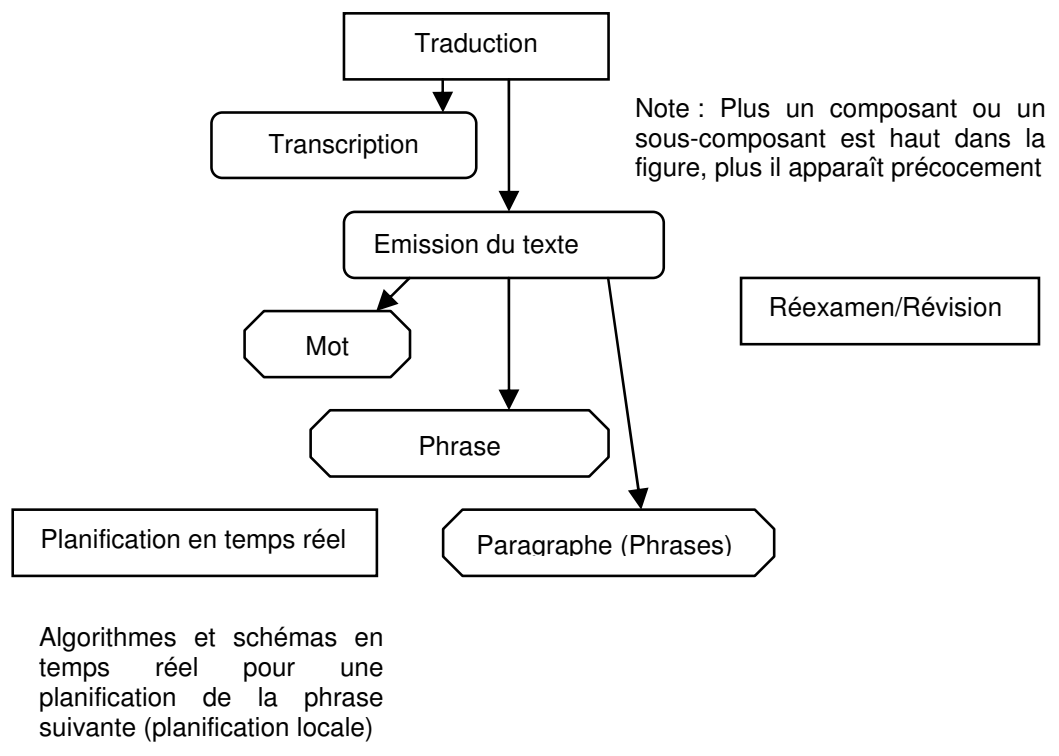

Figure 4.

Modèle du développement de la rédaction aux premiers niveaux scolaires (Emergence progressive des composants et sous-composants des processus)

d'après Berninger et Swanson (1994).

Pour les niveaux scolaires intermédiaires (cf. Figure 5), sous l'effet de l'âge (810 ans) et de la pratique, l'automatisation de la transcription graphique et orthographique favorise le développement d'une planification plus globale du contenu. La mobilisation du processus de révision reste délicate mais permet de gérer des erreurs plus diversifiées concernant différents niveaux de traitements langagiers (mots, phrases, ensemble du texte). 


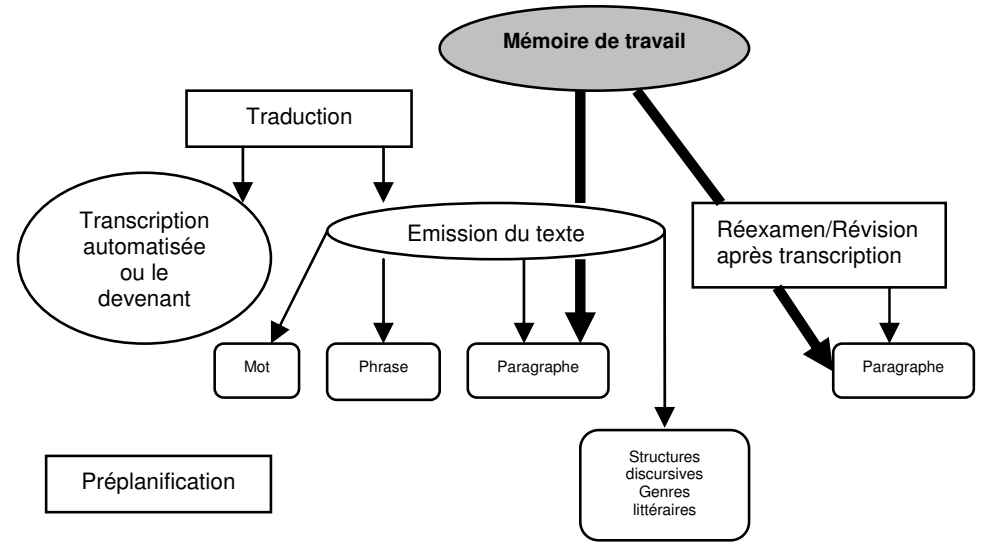

Figure 5.

Modèle du développement de la rédaction aux niveaux scolaires intermédiaires (Développement continu et émergence relative des composants et sous-composants des processus) d'après Berninger et Swanson (1994).

Ce n'est qu'au niveau du collège (11-12 ans; cf. Figure 6) que les processus déployés et mis en œuvre de façon efficace dans le cadre de la capacité de la mémoire de travail. La planification n'est pas encore suffisamment coordonnée avec les autres composants et ne le deviendra que plus tardivement (vers 16 ans).

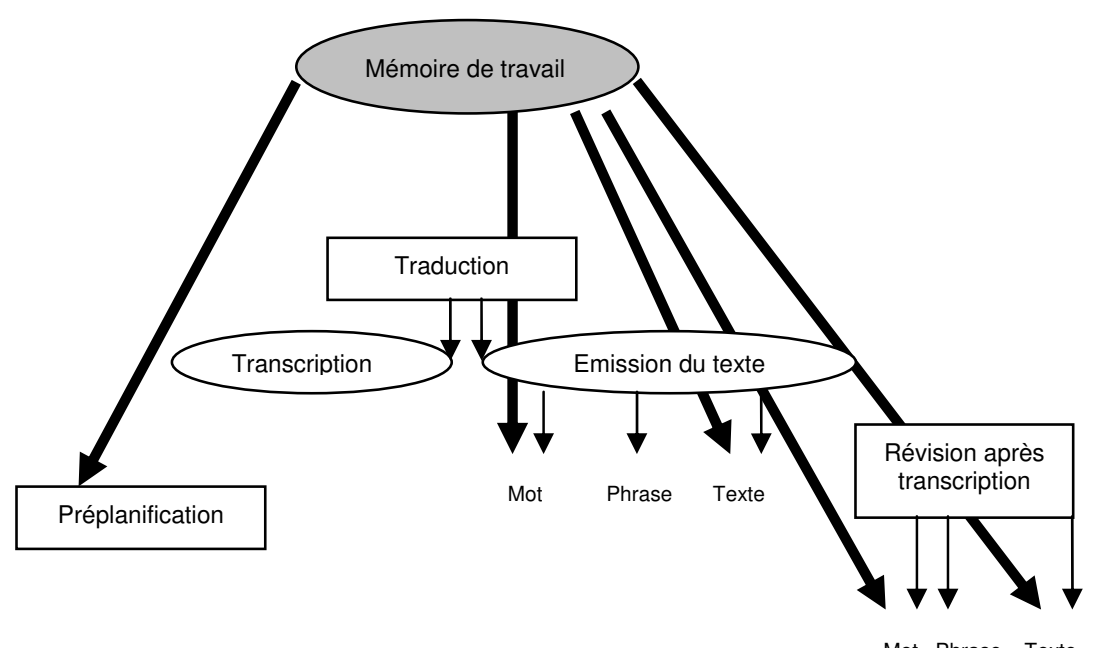

Figure 6. Modèle du développement de la rédaction aux niveaux scolaires élevés (Développement continu des composants des processus, de la mémoire de travail et de la métacognition à propos de la rédaction) d'après Berninger et Swanson (1994). 
A partir de ce modèle développemental, il n'est plus possible de considérer qu'un rédacteur expérimenté est celui qui a automatisé l'ensemble des opérations qu'il doit réaliser afin que son système cognitif ne soit pas surchargé par des traitements trop coûteux mettant à mal sa capacité mnésique limitée en ressources attentionnelles. Une fois que certaines opérations sont automatisées, plusieurs solutions fonctionnelles sont disponibles : un enchaînement successif et non parallèle de traitements ou un rallongement momentané du temps de traitement. Ce que soulignent ces modèles, c'est que certains traitements rédactionnels délibérés sont obligatoirement coûteux et que dans des situations de production délicate (par exemple, un manque de connaissances thématiques ou une difficulté à structurer un ensemble d'informations argumentatives, etc.), un rédacteur expérimenté peut délibérément leur attribuer encore plus de ressources afin d'être efficace.

\section{Modèles rédactionnels de l'adulte «composant" avec la mémoire de travail}

Il a fallu attendre une quinzaine d'années pour que la mémoire de travail soit clairement intégrée dans les architectures des composants utiles à la rédaction de textes chez le rédacteur adulte. Ainsi, dans le même temps et dans le même ouvrage, Hayes (1996) et Kellogg (1996) redéfinissent le champ de recherche, proposant alors des directions de travail à partir d'un modèle de plus en plus complexifié et intégratif de l'ensemble des déterminants de l'activité pour le premier, et d'un modèle élagué, simplifié et restreint à l'activité rédactionnelle stricto sensus pour le second. Dans les deux cas, la mémoire de travail devient un composant majeur de l'activité. Par ailleurs, en écho au modèle de Flower et al. (1986), Butterfield, Hacker et Alberston (1996) redéfinissent le contexte cognitif dans lequel le contrôle de la production écrite, c'està-dire la révision, peut être exercée.

Il n'est bien sûr pas possible de décrire par le menu ces architectures cognitives. Quelques informations justes utiles pour le propos de ce papier seront donc données ci-après.

\subsection{Hayes (1996) ne veut rien oublier}

Selon ses propres termes, Hayes (1996), propose un modèle individuoenvironnemental de la rédaction de textes. Cognition, affect et mémoire relèvent des caractéristiques de l'individu et les environnements social et physique permettent de caractériser la nature du contexte de production. Il illustre avec plusieurs résultats expérimentaux, les raisons de ces différents changements. L'architecture ainsi dessinée est alors très étendue (cf. Figure 7). 


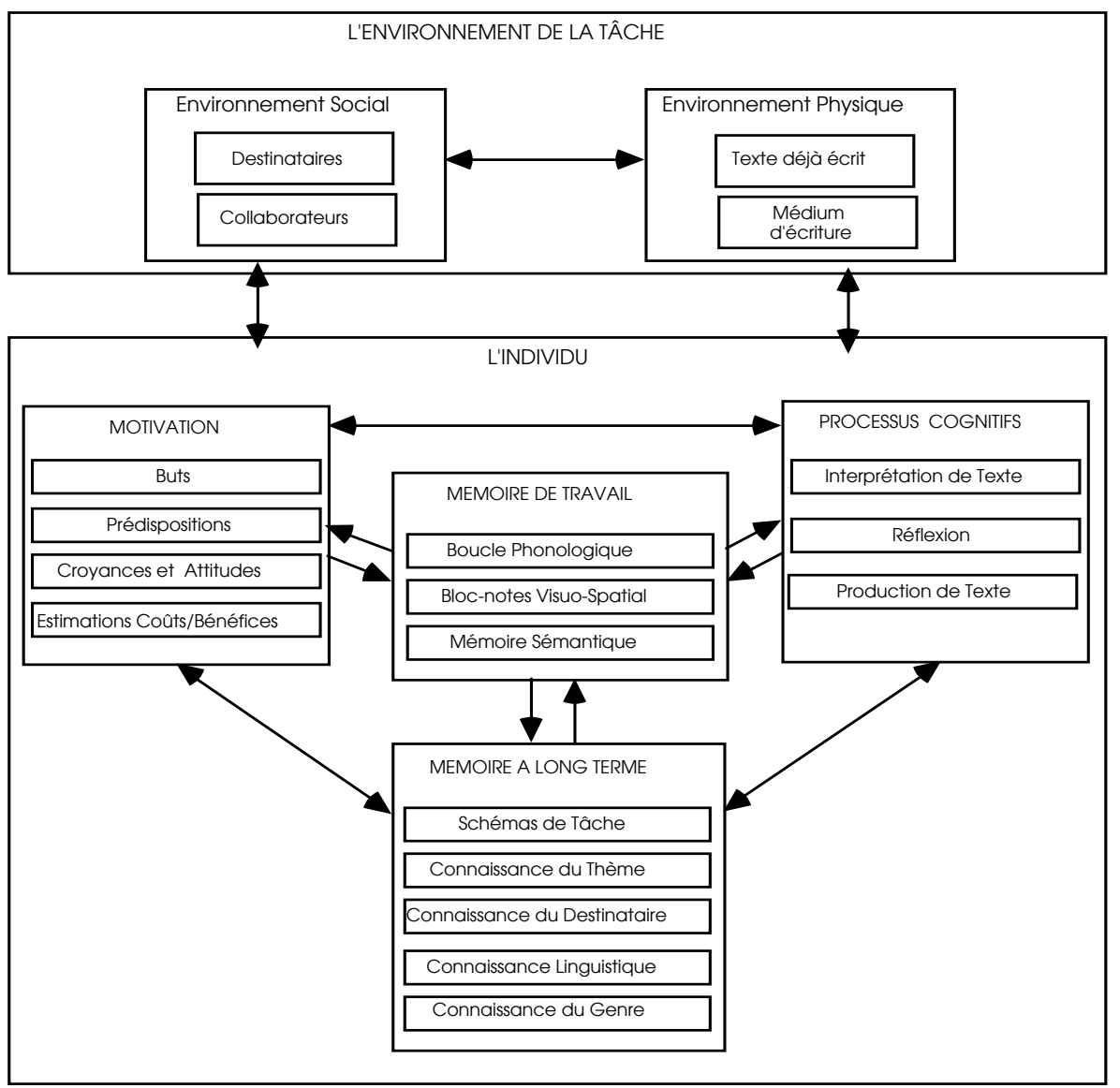

Figure 7.

Le «nouveau modèle » des processus rédactionnels selon Hayes (1996) permettant «d'intégrer cognition et affect».

Hayes (1996) propose quatre différences fondamentales d'avec son premier modèle : (1) L'accent doit être mis sur le rôle central de la mémoire de travail, intégrée d'ailleurs de façon centrale dans le schéma ; (2) En raison du formatage multimédia de plus en plus florissant des documents écrits, les représentations visuo-spatiales et linguistiques sont introduites ; (3) Une place significative est faite à la motivation et à l'affect qui jouent un rôle majeur dans la production écrite; (4) Les processus cognitifs (planification, génération de texte, révision) sont intégrés dans des structures cognitives plus générales qui partagent des opérations et des ressources cognitives (réflexion, production de texte, interprétation du texte). Tout en ayant réalisé une analyse très fine des différents types de planification (planification par abstraction, par analogie, par modélisation), Hayes (1996) insère cette activité dans le composant réflexion, dont le rôle est de transformer des représentations internes en d'autres représentations internes à l'aide d'outils cognitifs comme la résolution de problème, la prise de décision, la production d'inférences. La production de texte concerne, à la fois, la 
production verbale orale et écrite dont certaines opérations sont communes. Elle utilise les représentations internes issues de l'analyse du contexte afin d'aboutir à une sortie écrite, parlée ou graphique. L'interprétation de texte s'appuie sur la lecture, l'écoute et l'examen graphique pour créer des représentations internes à partir d'entrées linguistiques et graphiques. Ces représentations sont prises en charge par une structure de contrôle permettant via le composant de production, la transformation du texte.

Pour ce qui concerne la mémoire de travail, Hayes (1996) reprend le modèle de Baddeley (1990) dont il reproduit la structure, l'administrateur central partageant des fonctions exécutives avec le processus de réflexion. Toutefois, il y adjoint un stockage sémantique supplémentaire (mémoire sémantique) afin de maintenir les unités sémantiques inclues dans le message pré-verbal et exploitées lors de la génération du texte. Ce registre est très comparable au buffer épisodique proposé ultérieurement par Baddeley (2000) dans la dernière version de son modèle de la mémoire de travail. Enfin, si Hayes (1996) rappelle l'importance fonctionnelle de la boucle phonologique dans la mise en texte, il insiste sur celle du calepin visuo-spatial qui interviendrait aussi lorsque, à l'aide des processus de lecture et d'édition, le rédacteur révise son texte dont les particularités de mise en forme matérielle peuvent être utilisées.

\subsection{Kellogg (1996) simplifie mais spécifie}

Dans une synthèse très féconde sur la rédaction de textes, Kellogg (1994) rassemblait les théories sur l'activité rédactionnelle alors disponibles ainsi que ses propres acquis expérimentaux sur la mobilisation en temps réel des processus rédactionnels et l'effort cognitif (ou coût en ressources cognitives) associé à cette mobilisation. Il substitue à celui de Hayes et Flower (1980), un modèle qui n'a pas le succès escompté (cf. Figure 8). Comparativement au modèle princeps, deux composants sont introduits. Mémoire de travail et Mémoire à long terme sont différenciées. En faisant figurer sur le schéma la façon différente dont ces deux mémoires peuvent être mobilisées, Kellogg (1994) tente ainsi de rendre compte, chez l'adulte, de l'activation de l'une ou l'autre des stratégies rédactionnelles établies Scarmadalia et Bereiter (1991). Par ailleurs, et même si Kellogg (1994) fait explicitement référence à Baddeley (1986) en soulignant que la mémoire de travail offre un « espace de travail », celle-ci est reléguée au stockage des éléments en cours de traitement comme s'il s'agissait d'une mémoire à court terme assurant un stockage passif de schémas (séquences d'actions bien maîtrisées). Le fonctionnement du rédacteur serait alors sous la dépendance de ces schémas (schémas de phrase, de paragraphe, de document) activés automatiquement par des priorités internes et/ou des indices environnementaux qui ne feraient ainsi pas appel à une attention délibérée. En conséquence, Kellogg (1994) introduit aussi un composant attentionnel dont la fonction est d'attribuer volontairement du temps et des ressources attentionnelles aux différents traitements, temps et effort que Kellogg sait alors évaluer expérimentalement (pour un descriptif, voir Piolat \& Olive, 2000). 


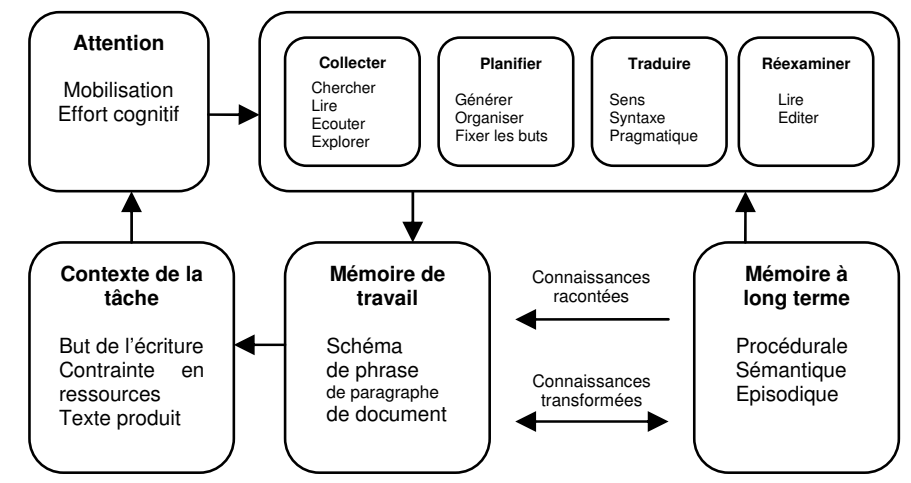

Figure 8.

Les composants cognitifs de l'habileté rédactionnelle d'après Kellogg (1994).

Le modèle de Kellogg (1994) constitue donc une «déroutante » interprétation de la mission que remplit la mémoire de travail selon Baddeley (1990) alors que ce dernier regroupe système attentionnel et mémoire de travail. La distinction entre mémoire de travail et composant attentionnel ne sera pas reprise par Kellogg dans son modèle de 1996, étayage majeur actuellement pour les recherches évaluant les relations entre les différents registres de la mémoire de travail et la rédaction de texte. L'architecture de 1996 (cf. Figure 9) est intéressante à deux titres : (a) le regroupement des processus rédactionnels proposés et (b) la façon dont ces processus exploitent (ou non) les ressources de la mémoire de travail.

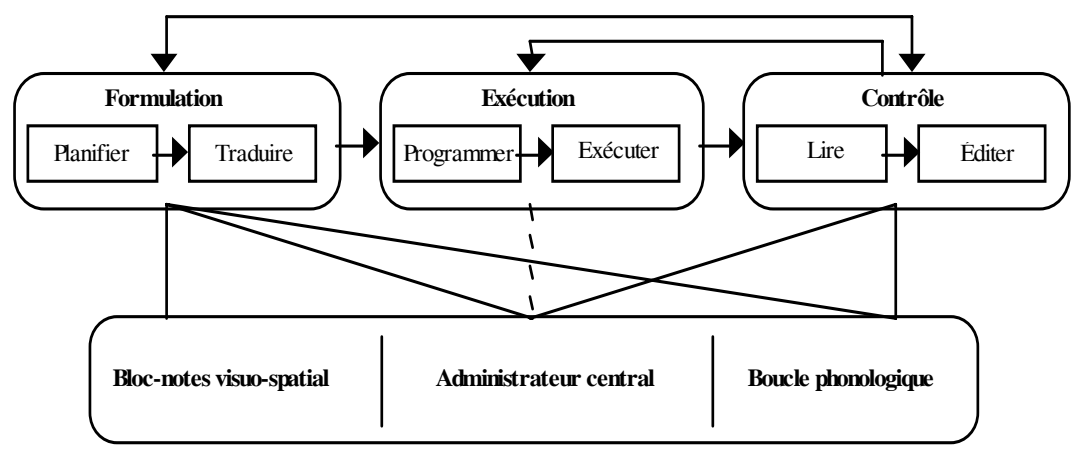

Figure 9. Les ressources de la mémoire de travail utilisées par les systèmes de formulation, d'exécution et de contrôle, selon Kellogg (1996).

Comparativement au modèle princeps de Hayes et Flower (1980) et à son propre modèle de 1994, un composant d'exécution est introduit pour marquer l'importance de la programmation et de la production grapho-motrice de l'écriture. La formulation prend en charge les aspects de planification et de mise en texte du message comme proposé dans le modèle princeps. Le contrôle permet de lire et vérifier le 
message en cours de composition et de l'éditer en diagnostiquant des erreurs et en les corrigeant si nécessaire. Pour l'essentiel, les caractéristiques fonctionnelles des processus et leurs interrelations sont du même ordre que celles de Hayes et Flower (1980). A l'exception de l'exécution (chez les adultes), les deux autres composants de la production de textes font largement appel à l'administrateur central, que cela soit pour coordonner les processus rédactionnels ou pour leur allouer des ressources cognitives. De plus, selon Kellogg (1996), pour planifier leur texte, les rédacteurs visualisent des images, des diagrammes et des plans. Aussi, la planification engagerait le calepin visuo-spatial comme la boucle phonologique, alors que les processus de traduction, de lecture et d'édition, engageraient uniquement la boucle phonologique. Ce modèle est heuristique comme l'a été, en son temps, celui de Hayes et Flower (1980). Aux chercheurs en psychologie cognitive de montrer la validité des hypothèses ainsi établies à l'aide des méthodes de double et triple tâche (Olive, 2002 ; Piolat \& Olive, 2000). Les travaux sont en cours.

\subsection{Butterfield, Hacker et Alberston (1996) complètent le processus de révision}

Butterfield et al. (1996) complètent des aspects restés implicites dans le modèle de révision de Flower et al. (1986) en distinguant clairement la mémoire à long terme et la mémoire de travail et, au sein de cette dernière, les niveaux cognitifs des niveaux métacognitifs. Ce modèle est évoqué ici car la capacité des rédacteurs à contrôler ce qu'ils font constitue une part importante de l'expertise rédactionnelle (Piolat, 1998).

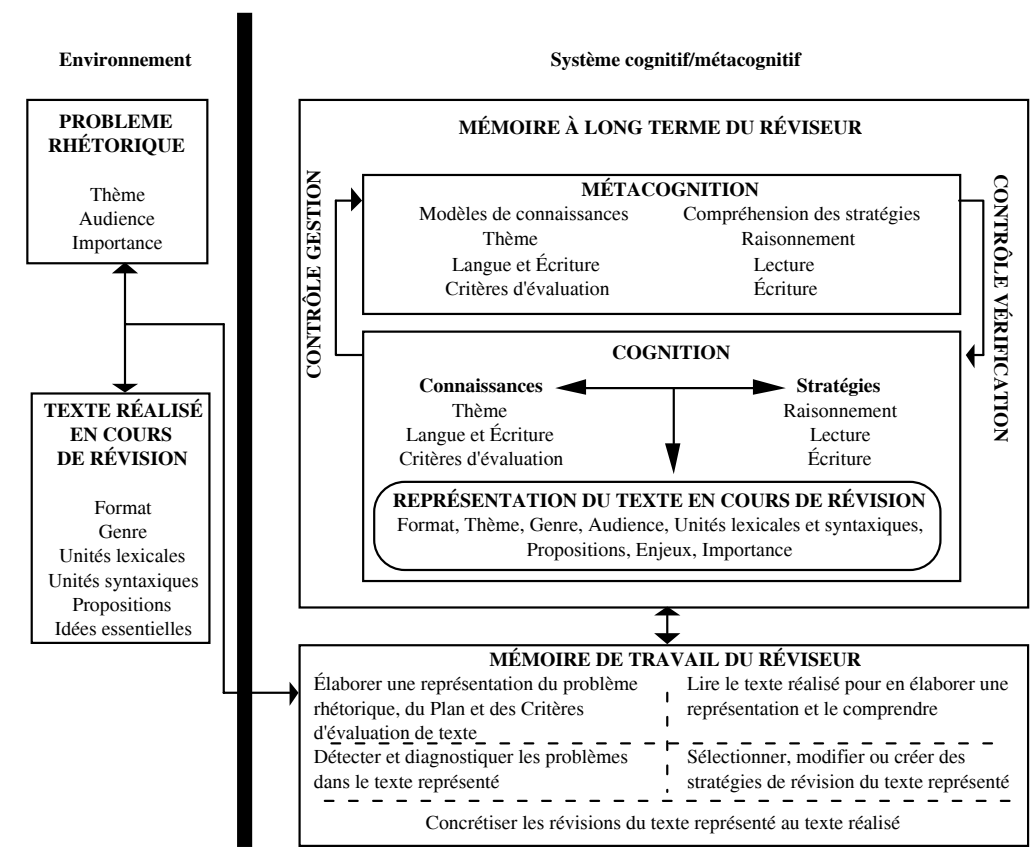

Figure 10.

Modèle procédural de révision d'après Butterfield, Hacker et Alberston (1996). 
Leur modèle de la révision de texte est constitué de deux composants (cf. Figure 10). L'environnement regroupe les contraintes conférant à la tâche d'écriture le caractère d'un problème rhétorique et le texte déjà réalisé à réviser. Le système cognitif/ métacognitif associe mémoire à long terme et mémoire de travail du réviseur. D’un point de vue fonctionnel, le support essentiel de la révision est la représentation mentale du texte à réviser élaborée en mémoire de travail, même si les modifications sont observables sur le texte déjà réalisé. Cette représentation dépend des contraintes prises en compte par le réviseur, de l'importance qu'il accorde à la révision (problème rhétorique) et des traitements cognitifs et métacognitifs que ce dernier met en oeuvre au cours de la lecture et de la révision du texte. Cette dernière est réalisée par les processus de représentation de la tâche, de représentation du texte, de détection et de diagnostic des problèmes du texte et de choix de stratégies de modification (Flower et al., 1986). Ces processus opèrent de façon délibérée et, de ce fait, interviennent en mémoire de travail. Les informations qu'ils traitent sont issues de l'environnement comme de la mémoire à long terme. La capacité limitée de la mémoire de travail contraint la révision, aussi l'allocation de ressources aux différents processus se fait sur un mode compétitif non prédéterminé. Les ressources peuvent être réparties entre tous les processus ou allouées à un seul, suivant les exigences du moment. Un transfert bidirectionnel d'informations entre mémoire de travail et mémoire à long terme permet de récupérer les connaissances nécessaires aux traitements et de renvoyer en mémoire à long terme du matériel déjà traité ou en cours de traitement jusqu'à une réutilisation éventuelle. Ce retour en mémoire à long terme (niveaux cognitif et métacognitif) permet de diminuer le coût cognitif en mémoire de travail. En effet, les traitements opérés sur les informations issues de l'environnement ou de la mémoire à long terme peuvent intervenir en mémoire de travail quand ils sont délibérés, mais aussi directement en mémoire à long terme quand ils sont automatisés. Le modèle d'origine de Flower et al. (1986) est ainsi complété en faisant une large place à la puissance opérative de la mémoire de travail.

\section{Conclusion : De l'intérêt de ces changements architecturaux}

En présentant aussi succinctement ces architectures cognitives, le risque est pris de ne pas rendre suffisamment compte de la façon dont les chercheurs en psychologie cognitive les utilisent pour poser des hypothèses et les tester expérimentalement. Aussi, le lecteur d'une autre discipline s'il est, par exemple, engagé dans une analyse de corpus écrits ou dans une élucidation des contextes favorisant les apprentissages en classe, peut, à juste titre, douter de l'intérêt de ces variations schématiques pour accroître l'intelligibilité de ce qu'est l'activité de rédaction de textes. L'illustration qui suit, concernant l'activité de prise de notes pourra peut-être raviver sa curiosité.

La prise de notes est une activité d'écriture incessamment utilisée dans le milieu scolaire et pourtant peu (ou pas) enseignée (Piolat \& Boch, 2004). Pourtant, jour après jour, l'élève stocke ce qui est professé mais il prépare aussi des exposés, des dissertations, résout des problèmes variés en prenant des notes. Par ailleurs, cette 
activité est très fréquente dans la vie quotidienne et dans de nombreux milieux professionnels. Il s'agit donc d'une écriture de la mémoire (stabilisation d'informations utiles par la suite) mais aussi du travail (accomplir une activité mentale ou diriger de futures actions ou décisions).

En termes de psychologie cognitive, la prise de notes (surtout lorsqu'il s'agit de stocker des informations justes entendues) implique la gestion simultanée des processus de compréhension (accès au contenu et sélection des informations) et de production (mise en forme de ce qui est transcrit à l'aide de procédés abréviatifs, de raccourcis syntaxiques, de paraphrases d'énoncés, et de mise en forme matérielle de ses notes), (pour une synthèse, Piolat, 2001 ; 2004). Le noteur est confronté à des problèmes de rapidité de traitement de l'information de divers ordres qui mettent à mal la capacité limitée de sa mémoire de travail. Tout en écrivant, il est soumit à la cadence de parole d'un conférencier (ou à celle des protagonistes d'une réunion) et subit une pression temporelle notable, car son écriture, même abréviée, reste lente à réaliser. Il doit maintenir en mémoire de travail des informations transitoires dont la gestion temporelle est complexe, car il coordonne les informations utiles à sa compréhension du message et celles utiles à ce qu'il produit par écrit. Ses notes, comme le montre l'analyse des corpus, ne consistent pas en une simple transcription linéaire et continue de ce qu'il entend. Plusieurs paramètres conditionnent cette gestion comme le contenu de l'enseignement, les indices fournis par le conférencier, les pratiques culturelles, le niveau d'habileté du noteur. Puisqu'il est expérimentalement possible de mesurer le coût cognitif de l'exercice de différentes activités complexes (apprendre, lire, noter, rédiger) dans lesquelles la mémoire de travail est la cheville ouvrière, il est intéressant de conclure sur les résultats expérimentaux suivants (Piolat, Roussey \& Barbier, 2003; Piolat, 2004). La prise de notes est une activité plus coûteuse que la lecture ou encore que l'apprentissage de listes de mots. Toutefois, elle s'avère moins exigeante en ressources attentionnelles que la production écrite d'un texte. La mobilisation des processus de planification et de révision contribue largement à la qualité du texte produit (Olive \& Piolat, 2004). Elle peut aussi provoquer la créativité de ce qui est écrit par élaboration d'idées nouvelles et formulations inattendues (Lubart, 2003).

$\mathrm{Si}$ les modélisations récentes de la rédaction de texte en psychologie cognitive ont permis - et permettront - d'identifier certains aspects fonctionnels de l'activité rédactionnelle, elles ne peuvent, en totalité, rendre compte de ce type de résultats. Il est donc essentiel de poursuivre, en la diversifiant, l'approche scientifique (modèles et expérimentations) afin d'approcher les relations entre rédaction de textes et affect comme le propose Hayes (1996), mais aussi sur celles entre rédaction et créativité. 


\section{RÉFÉRENCES}

Alamargot, D., \& Chanquoy, L. (2002) Les modèles de rédaction de textes. In M. Fayol (Ed.), Production du langage (pp. 45-65). Paris : Hermès Science Publications.

BAdDeley, A. D. (1990) Human memory: Theory and pratice. Hillsdale, NJ : Laurence Erlbaum Associates [traduction, 1993, La mémoire bumaine: théorie et pratique. Grenoble: Presses Universitaire de Grenoble].

BAdDeley, A. D. (2000) The episodic buffer : a new component of working memory? Trends in Cognitive Sciences, 4(11), 417-423.

Berninger, V.W., \& Swanson, H.L. (1994) Modification of the Hayes and Flower model to explain beginning and developing writing. In E. Butterfield (Ed.), Advances in Cognition and Educational Practice, Vol. 2 : Children's Writing : Toward a Process Theory of Development of Skilled Writing (pp. 57-82). Greenwich, CT : JAI Press.

Bourdin, B. (2002) Apprentissage de la gestion de la production et contraints de capacité. In M. Fayol (Ed.), Production du langage (pp. 149-169). Paris : Hermès Science Publications.

Butterfield, E. C., HACKer, D. J., \& Albertson, L. R. (1996) Environmental, cognitive, and metacognitive influences on text revision: Assessing the evidence. Educational Psychological Review, 8, 239-297.

Chanquoy, L., \& Alarmagot, D. (2002). Mémoire de travail et rédaction de textes: évolution des modèles et bilan des premiers travaux. L'Année Psychologique, 102, 363-398.

Dolchy, F. J., \& AleXAnder, P. A. (1995) Mapping prior knowledge: A framework for discussion among researchers. European Journal of Psychology of Education, 10(3), 225-242.

FAyOL, M. (1997) Des idées au texte. Psychologie cognitive de la production verbale, orale et écrite. Paris : P.U.F.

Flower, L. S., Hayes, J. R., Carey, L., Schriver, K., \& Stratman, J. (1986) Detection, diagnosis and the strategies of revision. College Composition and Communication, 37, 365387.

GAONAC'H, D., \& FrAdeT, A. (2003) La mémoire de travail : développement et implication dans les activités cognitives. In M. Kail \& M. Fayol (Eds.), Les sciences cognitives et l'école (pp. 91-150). Paris : PUF.

Gaonac'H, D., \& Larigauderie, P. (2000) Mémoire et fonctionnement cognitif. Paris : A. Colin.

Garcia-Debanc, C., \& Fayol, M. (2003) Apports et limites des modèles du processus rédactionel pour la didactique de la production écrite. Dialogue entre psycholinguistique et didacticien. Pratiques, 37-50.

HAYES, J. R. (1996) A new framework for understanding cognition and affect in writing. In C.M. Levy \& S. Ransdell (Eds.), The science of writing. Theories, methods, individual differences and applications (pp. 1- 27). Mahwah, NJ : L.E.A [traduction (1998). Un nouveau cadre pour intégrer cognition et affect dans la rédaction. In A. Piolat, \& A. Pélissier (Eds.), La rédaction de textes. Approche cognitive (pp. 51-101). Lausanne : Delachaux \& Niestlé]. 
HAYES, J. R., \& Flower, L. S. (1980) Identifying the organization of writing processes. In L. W. Gregg, \& E. R. Steinberg (Eds.), Cognitive processes in writing (pp. 3-30). Hillsdale, NJ : Lawrence Erlbaum.

KeLlogG, R. T. (1994) The Psychology of Writing. New York : Oxford University Press.

KeLLOGG, R. T. (1996) A model of working memory in writing. In M.C. Levy \& S.E. Ransdell (Eds.). The science of writing. Theories, Methods, Individual Differences and Applications (pp. $57-$ 71). Hillsdale, NJ : Laurence Erlbaum Associates [traduction (1998). Un modèle de la mémoire de travail dans la rédaction. In A. Piolat, \& A. Pélissier (Eds.), La rédaction de textes. Approche cognitive (pp. 103-135). Lausanne : Delachaux \& Niestlé].

LEVY, C. M., \& RANSDELL, S. E. (1995) Is writing as difficult as it seems? Memory and Cognition, 23(6), 767-779 [traduction (1998). Est-ce que la rédaction est aussi difficile qu'il y parait. In A. Piolat, \& A. Pélissier (Eds.), La rédaction de textes. Approche cognitive (pp. 137-181). Lausanne : Delachaux \& Niestlé].

Lubart, T. (2003) (Ed.). Psychologie de la créativité. Paris : A. Colin.

MCCuTCHen, D. (1996) A capacity theory of writing: Working memory in composition. Educational Psychology Review, 8(3), 299-325 [traduction (1998). Une théorie de la capacité pour la rédaction: la mémoire de travail dans la composqition. In A. Piolat, \& A. Pélissier (Eds.), La rédaction de textes. Approche cognitive (pp. 183-224). Lausanne: Delachaux \& Niestlé].

OLIVE, T. (2002) La gestion en temps réel de la production verbale : méthodes et données. In M. Fayol (Ed.), Production du langage (pp. 131-147). Paris : Hermès Science Publications.

Olive, T., \& Piolat, A., (2004) Activation des processus rédactionnels et qualité des textes. Le langage et l'bomme : logopédie, psychologie, audiologie (sous presse).

Piolat, A. (1990) Vers l'amélioration de la rédaction écrite. Apports des Technologies Nouvelles pour la Recherche et l'Apprentissage. Synthèse pour l'Habilitation à Diriger des Recherches, Université de Provence.

Piolat, A. (1998) Evaluation and Assessment of Written Texts. The Encyclopedia of Language and Education, Language Testing and Assessment (Vol.7, pp. 189-198), Dordrecht: Kluwer Academic Publishers.

Piolat, A. (1999) Planning and text quality among undergraduate students: Findings and questions. In M. Torrance, \& D. Galbraith (Eds.), Knowing what to write : Conceptual processes in text production (pp. 121-136). Amsterdam : Amsterdam University Press.

Piolat, A. (2001) La prise de notes. Paris : Presses Universitaires de France.

Piolat, A. (2004) La prise de notes: Écriture de l'urgence. In A. Piolat (Ed.). Ecriture. Approches en sciences cognitives. Aix-en-Provence : Presses Universitaires de Provence (sous presse).

Piolat, A., \& Boch, F. (2004) Apprendre en notant et apprendre à noter. In E. Gentaz, \& P. Dessus (Eds.), Comprendre les apprentissages. Psychologie cognitive et éducation (pp. 133-152). Paris : Dunod. 
Annie Piolat

Piolat, A., \& Olive, T. (2000) Comment étudier le coût et le déroulement de la rédaction de textes ? La méthode de la triple tâche: Un bilan méthodologique. L'Année Psychologique, 100, 465-502.

Piolat, A., \& PÉlissier, A. (1998) Etude de la rédaction de textes : contraintes théoriques et méthodes de recherches. In A. Piolat, \& A. Pélissier (Eds.), La rédaction de textes. Approche cognitive (pp. 225-269). Lausanne : Delachaux \& Niestlé.

Piolat, A., \& Roussey, J.-Y. (1992) Rédaction de textes. Éléments de psychologie cognitive. Langages, 106, 106-125.

Piolat, A., Roussey ; JY., \& BARbier, ML. (2003) Mesure de l'effort cognitif : Pourquoi est-il opportun de comparer la prise de notes à la rédaction, l'apprentissage et la lecture de divers documents? Arob@se, 1-2, 118-140 [http ://www.arobase.to].

Scardamalia, M., \& Bereiter, C. (1991) Literate expertise. In K.A. Ericsson \& J. Smith (Eds.), Toward a general theory of expertise (pp. 172-194). Cambridge: University Press [traduction (1998). L'expertise en lecture-rédaction. In A. Piolat, \& A. Pélissier (Eds.), La rédaction de textes. Approche cognitive (pp. 13-50). Lausanne : Delachaux \& Niestlé].

VAN WIJK, C. (1999) Conceptual processes in argumentation : a developmental perspective. In G. Rijlaarsdam, \& E. Espéret (Series Eds.), Studies in Writing (vol 4.), \& In M. Torrance, \& D. Galbraith (Vol. Eds.), Knowing what to write: conceptual processes in text production (pp. 31-50). Amsterdam : Amsterdam University Press.

NB : Les références de l'auteur sont téléchargeables à l'adresse suivante

[http ://www.up.univ-mrs.fr/wpsycle/membres/enseignants/apiolat.html] 